\title{
Simeon T. Cantril, M. D. 1908-1959
}

Dr. Simeon T. Cantril ist am 10. September 1959 im Alter von nur 51 Jahren an einer Coronarsklerose gestorben. SeinTodisteinschwerer Schlag fur die amerikanische Strahlentherapie.

Cantril begann seine Spezialausbildung als Assistent unter Dr. Max Cutler am Michael Reese Hospital in Chicago. Entscheidend wurde sein Werdegang beeinflufit wahrend seiner Lehrzeit unter Dr. Henri Coutard, zuerst im Institut Curie in Paris und dann im Chicago Tumor Institute. Im Jahre 1938 akzeptierte er die Stelle als Direktor des Tumor Instituts am Swedish Hospital in Seattle, die er bis zu seinem Tode inne hatte. Er wurde 1949 zum Associate Professor of Radiology an der neu organisierten Medical School der University of Washington ernannt. Necrologia

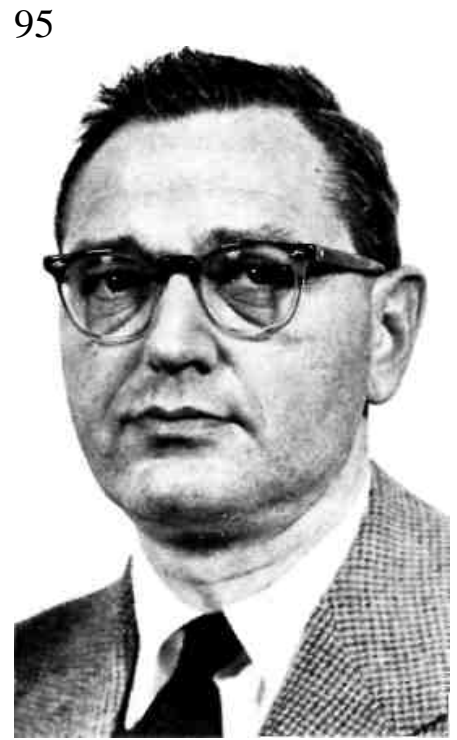

Cantril war Arzt im Grande seines Wesens. Medizin war fur ihn nicht Beruf, sondern Berufung und Lebensform. Der Kranke und nicht die Krankheit war fur ihn stets die Einheit. So war er hervor-ragend geeignet, Coutards geniale, aber nicht immer praktischen Ideen der klinischen Medizin nutzbar zu machen. Seine Fahigkeit, wissen-schaftliche Erkentnisse und technische Sorgfalt mit wahrem Arzttum zu vereinen, machte ihn zum wahrhaft groBen Kliniker, vergleichbar Klinikern von der Statur Osiers und Halsteds, die in dieser Generation technischer Spezialisten so selten geworden sind und die unter den Radiologen so wenige Vertreter haben. Seine wissenschaftlichen Veroffentlichungen auf dem Gebiete der Strahlentherapie, der Atommedizin und des Strahlenschutzes - in medizinischen Zeitschriften und Monographien sind in ihrer Grund-lichkeit und Zuverlassigkeit zu bekannt, um eine Aufzahlung hier zu erfordern. Er war stets bereit, unvoreingenommen neue Moglichkeiten und Methoden zu priifen, aber mit charakteristischem Realismus konzentrierte er sein Interesse auf die Behandlung der praktisch wichtigen Krebsformen, fur die die Strahlenbehandlung sich als die wertvollste 
Methode erwiesen hatte. So war er unermiidlich in seinen Bemuhungen, die Behandlung der Carcinome der Cervix und des Larynx zu verbessern, nicht durch technische Spitzfindigkeiten, son-

96

\section{Necrologia}

dern durch nie erlahmende Analyse und Kritik seiner eigenen Erfolge und MiBerfolge. Seine Monographic iiber das Cervixcarcinom und die groBe historisch-kritische Arbeit iiber das Carcinom des Larynx - sein wissenschaftliches Testament und sein letzter Tribut fur seinen bewunderten Meister Coutard - legen davon Zeugnis ab. Die Janeway Lecture in 1957 iiber «Die Beitrage der Biologie zur Strahlentherapie» zeugt fur sein besonderes Talent, Probleme in richtiger Perspektive zu sehen.

Cantril erkannte friih das Ziel seines beruflichen Lebens und blieb konsequent in seiner Verfolgung: die Organisation eines Pionier-instituts, in dem Strahlentherapie des Krebses als klinische Kunst geiibt und gelehrt werden konnte. Er verstand, daB die ungewohnliche Kombination der im Swedish Hospital verfiigbaren technischen Appa-ratur - die damals nirgendswo anders im Lande vergleichbar war -mit der Unabhangigkeit, die die verstandnisvolle Arzteschaft und die Verwaltung des Krankenhauses ihm dort gewahrten, bessere Moglich-keiten boten, sein Ziel zu erreichen, als beruhmtere Institute mit langbestehender Tradition. Er hat deshalb mehrere ehrenvolle Be-rufungen in den folgenden Jahren abgelehnt. Es ist ihm so durch seine eigene zielbewuBte und unermudliche Arbeit gelungen, ein Institut zu schaffen, das als fuhrendes Behandlungs- und Unterrichtszentrum anerkannt ist. Cantril selbst wiirde wahrscheinlich die Griindung seiner Schule klinischer Strahlentherapeuten als seinen wertvollsten Beitrag und ihre Weiterentwicklung durch seine Mitarbeiter und Schiller als sein ehrenvollstes Andenken betrachten.

Wahrend des Krieges wurde er - zusammen mit Dr. Robert S. Stone -mit der Uberwachung der Gesundheits- und Strahlenschutzprobleme fiir das Personal des Atombombenprojekts betraut. Mit charakte-ristischer Griindlichkeit und Weitblick behandelte er die neuen Probleme, die mit den damals im wesentlichen unbekannten Risiken verbunden waren. Diese Untersuchungen, die sich mit toxikologischen und Strahlenschadigungen befassen, sind nach ihrer Deklassifikation in einer Reihe von grundlegenden Arbeiten veroffentlicht worden, die heute fundamentale Informationen von unschatzbarem Werte ent-halten.

Cantril blieb nach dem Kriege eng verbunden mit dem sich schnell entwickelnden Gebiet der Atommedizin und wurde als Autoritat anerkannt. Es ist vielleicht gerade diese griindliche Sachkenntnis, die ihn abhielt, sich von der Welle modisclier Begeisterung zu billiger Beruhmtheit tragen zu lassen, wie er auch einer ahnlichen Versuchung Necrologia

97

wahrend der Friihzeit der Supervolttherapie widerstanden hat. Er erkannte friih den wahren Platz der Isotopen in der Medizin und betonte die Grenzen ihrer praktischen Anwendbarkeit in der Behand-lung von Krankheiten. In seiner zusammenfassenden Arbeit iiber den «Gebrauch der radioaktiven Isotope in Biologie und Medizin» schloB er schon 1946, «wahrend unserer Lebenszeit sehe ich nicht das Millenium, in dem Hippokrates mit einem radioaktiven Caduceus Wunder der Heilung ausfuhren wird». Dieser Uberzeugung getreu hat er eine Isotopen-Abteilung im Tumorinstitut erst mehrere Jahre spater organisiert, als er selbst von der Art der Organisation iiberzeugt war, die fur klinischen Gebrauch notwendig war. 
Cantril reprasentierte als Mann und als Arzt alles was nobel ist in amerikanischer Medizin und im amerikanischen Leben. In Tun und Denken war er ein wahrer Demokrat, der sein ganzes Leben lang seinem Ideal, Abraham Lincoln, folgte. Intellektuelle Integritat und kompromiBlose Ehrlichkeit gegen sich selbst und andere, warmes, aber nie sentimentales Verstandnis fur seine Mitmenschen charakteri-sierten seine Personlichkeit. Seine weite Allgemeinbildung, sein Interesse an Literatur, Geschichte, Sprachen und Musik, und seine verstandnisvolle Achtung vor den europaischen Beitragen zur Zivili-sation, Medizin und Radiologie, verbunden mit seiner pragmatischen Lebensphilosophie, machten ihn zu einem wahren amerikanischen Humanisten. Durch seinen personlichen EinfluB hat Cantril vielleicht mehr als irgend ein anderer zur Entwicklung der mehr klinischen Gesamtein-stellung der Strahlentherapie in den Vereinigten Staaten beigetragen, die sich langsam durchzusetzen beginnt und die zu ihrer zunehmenden Anerkennung als selbstandiges Spezialfach und zu ihrem groBeren Ansehen unter der Arzteschaft gefiihrt hat. Cantrils nationales und internationales Ansehen fand formale Anerkennung in seiner ein-stimmigen Wahl zum Founding President des neu gegriindeten American Club of Therapeutic Radiologists bei der Eroffnungssitzung im Jahre 1958 und seiner Wahl zum Executive Secretary for North America, als der International Club of Radiotherapists 1952 ge-griindet wurde.

Wir alle, denen der Fortschritt dieser noch kampfenden Spezialitat der Strahlentherapie am Herzen liegt, sind uns der GroBe des Ver-lustes bewuBt. «Das ist ein harter, bitterer Abscbied, den wir in dieser Stunde nehmen. Wir miissen uns trennen - im Raum und in der Zeit, wenn auch nicht im Geiste - von einem seltenen Menschen...» Aber

8 Oueologia, Vol. 13, No. $1(1 \% 0)$

98

Necrologia

die Erinnerung an dieses noble Leben, an den Mann und den Arzt, wird fortdauern und ihren EinfluB ausiiben auf seine Mitarbeiter, auf seine Schiiler und seine vielen Freunde als eine lebendige Quelle der Inspiration.

«Du hast ein herrlich Werk vollbracht,

Wie dank ich Deiner klugen Macht.»

Franz Buschke (San Francisco, Calif.)

Varia

Beschliisse des IX. Internationalen Kongresses fiir Radiologie, Miinchen 1959

Der IX. Internationale KongreB fiir Radiologie (I. C. R.), der vom 23. bis 30. Juli 1959 in Miinchen tagte, hat folgende Beschliisse gefafit, die ihm von der Internationalen Kommission fiir Stadieneinteilung und Erfolgsstatistik (I.C.P.R.) unter dem Prasidium von Prof. Dr. H. R. Schinz, Ziirich, vorgelegt wurden:

Eine internationale klinische Stadieneinteilung der Tumoren ist dringend notwendig. Das 1953 vom VII. Internationalen Kongrefi fiir Radiologie durch das I. C. P. R. unter dem Vorsitz von Prof. Dr. Heyman, Schweden, vorgelegte und vom KongreB genehmigte TNMSystem ist heute allgemein angenommen und wurde zuerst beim Brust- und Larynxkrebs angewandt. In Zukunft wird es analog beim Krebs der natiirlichen Korperoffnungen, bei den metastasierenden Geschwiilsten der Haut und beim Bronchus-Carcinom zur Anwendung kommen.

Fiir die Malignome der Gebarmutter, fiir die intestinalen Krebse und fiir die Krebse der Gallenblase, des Pankreas, der Nieren und der Harnblase empfiehlt sich die Stadieneinteilung nach dem TM-System. 
Bei den nicht metastasierenden (semimalignen) Geschwiilsten empfiehlt sich die Stadieneinteilung nach dem T-System.

Bei unbekanntem Primartumor gibt es nur das M-System.

Wiinschbar fiir die Zukunft ist eine engere Zusammenarbeit zwischen der I. C. P. R. und dem Comite de nomenclature et de statistique de TO. I. C. C, den Ver-tretern der Internationalen Chirurgen- und Gynakologen-Vereinigungen und der Organisation Mondiale de la Sante (O. M. S.).

ImNamenderl.C.P.R.

Der Prasident:

Miinchen, den 30. Juli 1959 Prof. Dr. H. R. Schinz

Decisions of the International Congress of Radiology 19S9

The IXth International Congress of Radiology which has been in session from July the 23rd to July the 30th 1959 in Munich, has taken the following decisions 